\title{
Deuteron nuclear data for the design of accelerator-based neutron sources: Measurement, model analysis, evaluation, and application
}

\author{
Yukinobu Watanabe ${ }^{1, \text { a }}$, Tadahiro Kin ${ }^{1}$, Shouhei Araki ${ }^{1}$, Shinsuke Nakayama ${ }^{2}$, and Osamu Iwamoto ${ }^{2}$ \\ ${ }^{1}$ Depertment of Advanced Energy Engineering Science, Kyushu University, 6-1 Kasuga-koen, Kasuga, Fukuoka 816-8580, Japan \\ 2 Nuclear Science and Engineering Center, Japan Atomic Energy Agency, 2-4 Shirakata, Tokai, Naka, Ibaraki 319-1195, Japan
}

\begin{abstract}
A comprehensive research program on deuteron nuclear data motivated by development of accelerator-based neutron sources is being executed. It is composed of measurements of neutron and gammaray yields and production cross sections, modelling of deuteron-induced reactions and code development, nuclear data evaluation and benchmark test, and its application to medical radioisotopes production. The goal of this program is to develop a state-of-the-art deuteron nuclear data library up to $200 \mathrm{MeV}$ which will be useful for the design of future $(d, x n)$ neutron sources. The current status and future plan are reviewed.
\end{abstract}

\section{Introduction}

In recent years, intensive accelerator-based neutron sources with deuteron-induced reactions on light elements such as $\mathrm{Li}, \mathrm{Be}$, and $\mathrm{C}$ have been proposed for various neutron beam applications, e.g., production of medical radioisotopes [1], boron neutron capture therapy [2], irradiation testing of fusion reactor materials [3], production of high intensity RI beams of neutron-rich nuclei [4], and transmutation of long-lived radioactive nuclear waste [5]. The research and development of such neutron sources has led to the revival and increasing interest on the study of deuteron-induced reactions.

Figure 1 shows measured double-differential thick target neutron yields (TTYs) of $(p, x n)$ and $(d, x n)$ reactions on ${ }^{9} \mathrm{Be}$ at $40 \mathrm{MeV}[6,7]$ and $(d, x n)$ angular distribution at $13.5 \mathrm{MeV}$ [8]. From the viewpoint of production of mono-energetic neutrons, the $(p, x n)$ reaction is known to be superior to the $(d, x n)$ reaction. However, neutrons generated from deuteron breakup reactions have some advantages, i.e., more intensive neutron yields and characteristic broad peak structure around half the incident energy as shown in Fig. 1(a). In addition, deuteron breakup reactions result in highly-focusing neutron beams with strongly forward peaked angular distribution as shown in Fig. 1(b). These features will be beneficial in the abovementioned applications.

The design of $(d, x n)$ neutron sources requires reliable knowledge about not only the interaction of deuterons with neutron converter material but also various nuclear reactions due to deuteron beam loss in the beam transport system. Therefore, comprehensive nuclear data of deuteron-induced reactions are indispensable over wide ranges of incident energy and target element for accurate estimation of neutron yields from the converter and induced radioactivity in constituent materials.

a e-mail: watanabe@aees.kyushu-u.ac.jp
An evaluated nuclear data library called TENDL [9] is available for deuteron-induced reactions up to $200 \mathrm{MeV}$, and a part of them has been stored in FENDL3 [10]. The deuteron TENDL library produced by the theoretical model code TALYS [11] has not yet been wellvalidated for available experimental data. Recent work with MCUNED by Sauvan et al. [12] revealed considerable underestimation of neutron production from thick copper target at forward angles.

Double-differential cross sections (DDXs) of neutron production are necessary for characterization of neutron sources and shielding design. However, experimental DDX data are very limited and no data exist above $50 \mathrm{MeV}$ [13]. Meanwhile, thick target neutron yield (TTNY) data are available for deuteron energies up to $65 \mathrm{MeV}$ [13] and there are a variety of measured $(d, x p)$ spectra over a wide range of target element for incident energies $\leq 100 \mathrm{MeV}$. Various activation cross sections for deuteroninduced reactions are compiled in Ref. [13].

Theoretical model calculations play an essential role in nuclear data evaluation in the case where available experimental data are not enough for the requirement. Particularly, theoretical models capable of describing deuteron breakup reactions well are required. More recently, model analyses of inclusive $(d, x p)$ reactions have been reported with attention to elastic and nonelastic deuteron breakup processes by several research groups [14-16]. We have been developing a code system dedicated for deuteron-induced reactions, called DEUteron-induced Reaction Analysis Code System (DEURACS), including pre-equilibrium and evaporation processes. It has been applied successfully to analyses of both inclusive $(d, x p)$ and $(d, x n)$ reactions [17-20] (and references therein). In addition, Avrigeanu et al. have intensively performed a series of their model analyses of activation cross sections [21]. Also, a Monte Carlo simulation to describe $(d, x n)$ reactions by combining the Intra-Nuclear Cascade of Liège (INCL) [22] and Distorted 

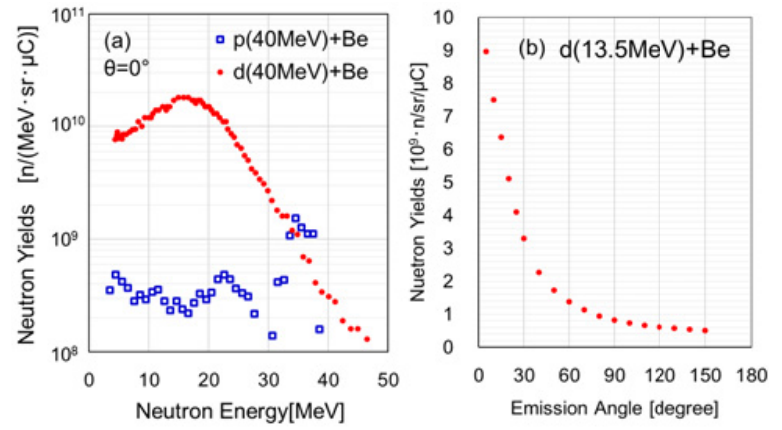

Figure 1. Measured neutron production yields: (a) p- and d-incidence on $\mathrm{Be}$ at $40 \mathrm{MeV}$ taken from Refs. [6,7], (b) d-incidence on Be at 13.5 MeV taken from Ref. [8].

Wave Born Approximation (DWBA) are available in the Particle and Heavy Ion Transport code System (PHITS) $[23,24]$.

Under these circumstances, we have launched a comprehensive research program on deuteron nuclear data, which is composed of measurements of neutron and gamma production DDXs and TTYs at the RCNP in Osaka University and the Kyushu University Tandem Laboratory, development of dedicated model code system and analyses of the measured data, cross section evaluation and benchmark test, and its application to medical radioisotopes production. Our goal is to develop a stateof-the-art deuteron nuclear data library up to $200 \mathrm{MeV}$ necessary for the design of deuteron accelerator-based neutron sources. The current status and future plan are presented in the following sections.

\section{Measurements}

\subsection{Thick target neutron yields at deuteron energies below $10 \mathrm{MeV}$}

We have carried out a series of measurements of doubledifferential neutron yields from thick targets irradiated by deuterons at the Kyushu University Tandem accelerator Laboratory (KUTL) [25-30]: carbon, aluminum, titanium, copper, niobium, and tantalum for bombardment energies of 5 and $9 \mathrm{MeV}$. In addition, double-differential thick target gamma-ray yields from carbon and aluminum irradiated by 5 and $9 \mathrm{MeV}$ deuterons were measured at the same time. The details of the experimental set up and procedure have been reported in Refs. [25-30].

A deuteron beam accelerated up to 5 or $9 \mathrm{MeV}$ was delivered to an irradiation target with stopping length in a compact vacuum chamber. Emitted neutrons were detected by an NE213 liquid organic scintillator $50.4 \mathrm{~mm}$ thick and $50.4 \mathrm{~mm}$ in diameter coupled optically with a Hamamatsu H6410 photomultiplier. The detector was placed in the distances from 1.6 to $2.4 \mathrm{~m}$ from the center of the target. Neutron yields from the target were measured at nine angles of $0^{\circ}, 15^{\circ}, 30^{\circ}, 45^{\circ}, 75^{\circ}, 90^{\circ}, 120^{\circ}$ and $140^{\circ}$ by changing the detector position. A background measurement with an iron shadow bar $150 \mathrm{~mm} \times 150 \mathrm{~mm}$ $\times 300 \mathrm{~mm}$ thick placed between the target and the neutron detector was carried out at each angle in order to estimate the contribution of background neutrons scattered from the floor and walls in the experimental room.

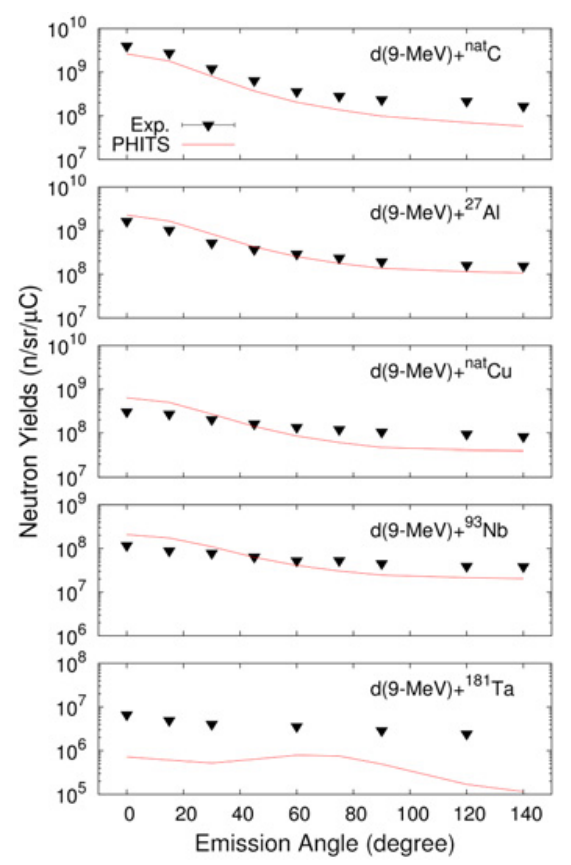

Figure 2. Comparisons of measured angular distributions of neutron yields integrated over emission energy above $2 \mathrm{MeV}$ at $9 \mathrm{MeV}$ with PHITS calculations using INCL4.6 + GEM model.

Finally, neutron energy spectrum was derived from light output spectrum by means of an unfolding method using the FORIST code [31] with the response function of the NE213 scintillator calculated by SCINFUL-QMD code [32]. In the similar way, the gamma-ray energy spectra for $\mathrm{C}$ and $\mathrm{Al}$ were obtained by an unfolding method using the FORIST code with the response functions of the NE213 detector calculated by EGS5 incorporated in PHITS code [23].

Figure 2 shows measured angular distributions of neutron yields integrated over emission energy above $2 \mathrm{MeV}$ at the incident energy of $9 \mathrm{MeV}$. Forward-peaked angular distribution is observed for each target, and the trend becomes prominent with decreasing target atomic number. The solid lines denote PHITS calculations with a combination of INCL4.6 [22] for the dynamical process and generalized evaporation model (GEM) [33] for the statistical decay process. The PHITS calculation exhibits forward-peaked angular distribution except for Ta. The calculation tends to overestimate the experimental neutron yields at angles less than $30^{\circ}$ except for $\mathrm{C}$ and $\mathrm{Ta}$ and underestimate them at larger angles. The results of $\mathrm{C}$ and Ta underestimate the measured data over the whole angular range. Since the intra-nuclear cascade model works well at high incident energies, its application to $9-\mathrm{MeV}$ deuteron incidence might be beyond the applicable scope of the model.

Next, the measured gamma-ray spectrum for carbon at $0^{\circ}$ is shown in Fig. 3. Three pronounced peaks are observed. They correspond to gamma-ray transitions between discrete levels in residual nuclei. Note that the measured angular distribution was almost isotropic for the incident energies of 5 and $9 \mathrm{MeV}$. The PHITS calculation using a combination with INCL, GEM, and EBITEM [34] models reproduces the spectral shapes and angular distributions generally well, although it underestimates the absolute gamma-ray yield by about $20 \%$. 


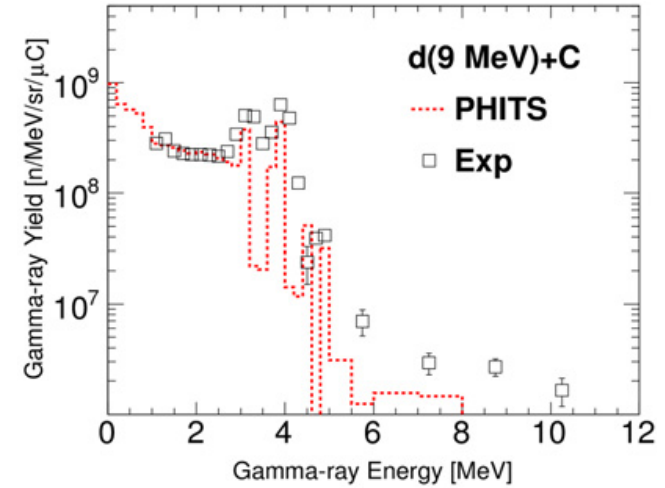

Figure 3. Measured gamma-ray yield at $0^{\circ}$ from carbon bombarded by $9-\mathrm{MeV}$ deuterons and PHITS calculation with INCL4.6 + GEM + EBITEM model.

\subsection{Double-differential neutron production cross sections in the 100 to $200 \mathrm{MeV}$ range}

We have measured double-differential neutron production cross sections (DDXs) for $102-\mathrm{MeV}$ deuteron induced reactions on ${ }^{\text {nat }} \mathrm{Li},{ }^{9} \mathrm{Be}$, ${ }^{\text {nat }} \mathrm{C},{ }^{27} \mathrm{Al}$, ${ }^{\text {nat }} \mathrm{Cu}$, and ${ }^{93} \mathrm{Nb}$ at forward angles $\left(\leq 25^{\circ}\right)$ by means of the time of flight (TOF) method with NE213 liquid organic scintillators at the Research Center of Nuclear Physics (RCNP), Osaka University [35].

A deuteron beam was accelerated up to $102 \mathrm{MeV}$ by the ring cyclotron, and pulsed at $1.09 \mathrm{MHz}$ using a beam chopper. The deuteron beam was delivered on a target placed in the beam swinger magnet and then transported to a Faraday cup in a beam dump. The beam current was changed from 5 to $60 \mathrm{nA}$, depending on target and measurement angle.

Energy spectra of neutrons emitted from the target were measured at angles of $0^{\circ}, 5^{\circ}, 10^{\circ}, 15^{\circ}, 20^{\circ}$ and $25^{\circ}$ for $\mathrm{Be}, \mathrm{C}, \mathrm{Al}\left(0^{\circ}\right.$ and $10^{\circ}$ for $\mathrm{Li}, \mathrm{Cu}$, and $\left.\mathrm{Nb}\right)$, by moving the target along the beam trajectory in the swinger magnet. The emitted neutrons were transported to the 100-m TOF tunnel after passing a movable collimator. A clearing magnet was installed in the collimator to remove background components of charged particles.

The energies of emitted neutrons were measured by the TOF method with two different sizes of NE213 liquid organic scintillators $(5.08 \mathrm{~cm}$ by $5.08 \mathrm{~cm}$ and $12.7 \mathrm{~cm}$ by $12.7 \mathrm{~cm}$ in diameter and length) coupled to photomultiplier tubes (hereafter referred to as 5.08-cm and 12.7-cm NE213 detectors, respectively). The $5.08-\mathrm{cm}$ and the $12.7-\mathrm{cm}$ NE213 detectors were placed at $7 \mathrm{~m}$ and $17 \mathrm{~m}$ from the target, respectively. Since the magnetic field of the clearing magnet influenced the photomultiplier tube placed at $7 \mathrm{~m}$, the magnet was switched off and a $5 \mathrm{~mm}$ thick NE102A plastic scintillator was employed as a veto detector of background charged particles in front of the $5.08-\mathrm{cm}$ NE213 detector.

The energy spectrum was converted to DDXs using the neutron detection efficiency, the solid angle and the deuteron beam current. The efficiency was calculated by the SCINFUL-QMD code [32]. The attenuation of neutron fluxes at 7 and $17 \mathrm{~m}$ through air was estimated by PHITS simulation, and the measured neutron flux was corrected by the estimated attenuation fraction. Finally, the two DDXs measured at $7 \mathrm{~m}$ and $17 \mathrm{~m}$ were merged at $15 \mathrm{MeV}$,
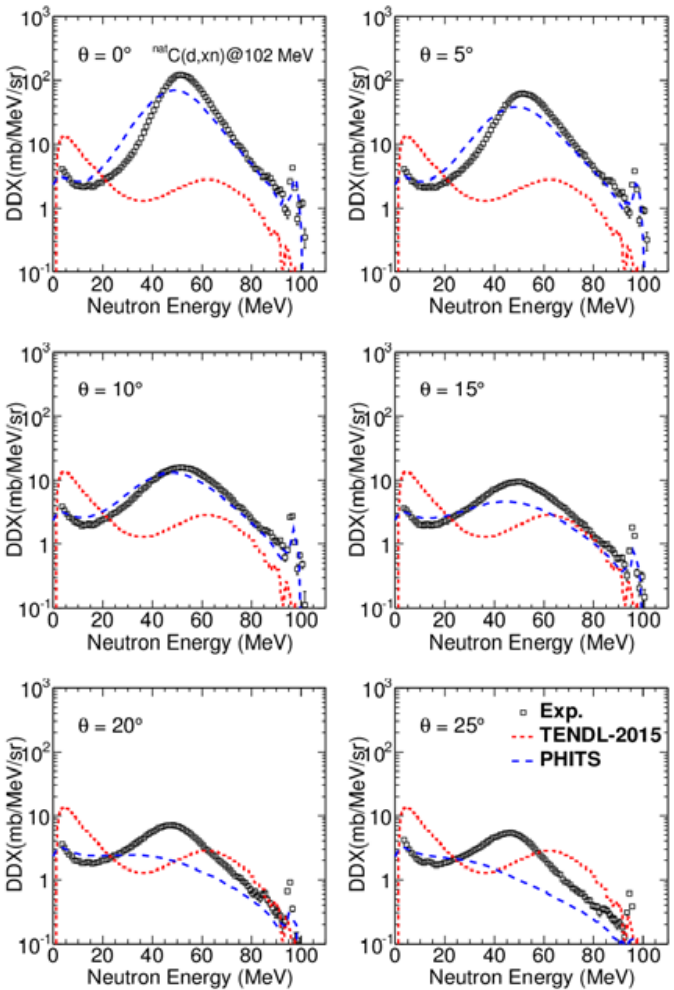

Figure 4. Comparison of measured double-differential cross sections of neutron production from carbon by $102-\mathrm{MeV}$ deuteron incidence with TENDL-2015 and PHITS calculation.

that is, the DDXs measured at $7 \mathrm{~m}$ was adopted in the lower energy range $(\leq 15 \mathrm{MeV})$ and the one measured at $17 \mathrm{~m}$ was done above $15 \mathrm{MeV}$.

The measured DDXs of the $\mathrm{C}(d, x n)$ reaction at $102 \mathrm{MeV}$ are shown by open squares in Fig 4. Note that the error bars indicate only the statistical error. The systematic error was estimated to be $11 \%$ to $12 \%$. A broad peak around half the incident energy and sharp peaks around $100 \mathrm{MeV}$ are observed in all the measured energy spectra. The height of the broad peak decreases rapidly with increasing angle. The broad peak may correspond to neutron emission via elastic and nonelastic breakup reactions, since the deuteron is a very loosely bound system of a proton and a neutron. The sharp peaks around $100 \mathrm{MeV}$ arise from single-proton transfer ${ }^{12} \mathrm{C}(d, n)$ reactions to discrete levels in the residual nucleus ${ }^{13} \mathrm{~N}$.

In Fig. 4, the experimental DDXs of ${ }^{\text {nat }} \mathrm{C}$ are compared with TENDL-2015 nuclear data and PHITS calculation in which INCL4.6 [22] and GEM [33] were used for description of the dynamical and subsequent evaporation processes and the KUROTAMA model [36] was adopted for calculation of the total reaction cross section. TENDL2015 overestimates the low-energy evaporation component at whole angles, and cannot reproduce the observation that the broad peak around $50 \mathrm{MeV}$ decreases rapidly with increasing emission angle. Meanwhile, the PHITS calculation shows overall agreement with the experimental DDXs at angles below $10^{\circ}$, although the calculated peak around $50 \mathrm{MeV}$ is slightly wider than the experimental one. Moreover, the PHITS calculation cannot reproduce the spectral shape of the observed broad peak component at larger angles $\left(\geq 15^{\circ}\right)$. 


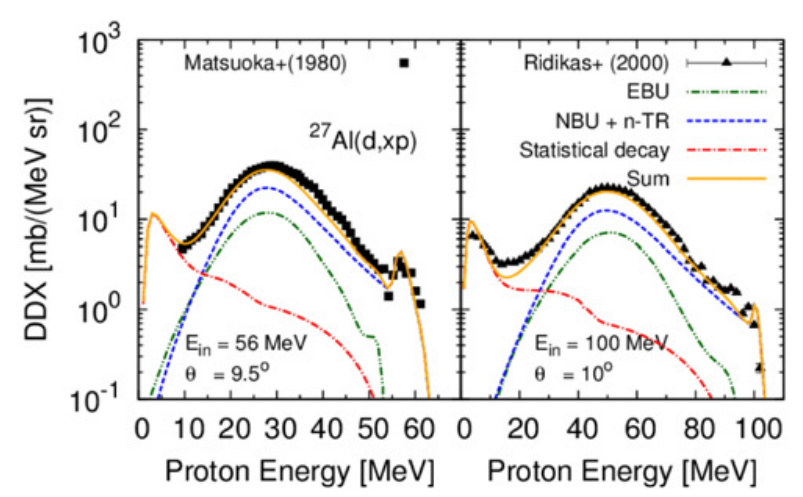

Figure 5. Calculated and experimental DDXs of the ${ }^{27} \mathrm{Al}(d, x p)$ reactions at 56 and $100 \mathrm{MeV}$.

In the future, we plan to perform a similar measurement at $200 \mathrm{MeV}$ to investigate the incident energy dependence of neutron production in deuteron-induced reactions.

\section{Model analysis and nuclear data evaluation}

We have been studying a model calculation method capable of describing inclusive nucleon emission quantitatively well [17-20,37-39]. The model calculation uses the continuum discretized coupled channels (CDCC) method for elastic breakup (EBU), the Glauber model for nonelastic breakup (NBU), a zero-range DWBA approach for single-nucleon transfer (TR) to bound states in the residual nuclei, and the exciton plus Hauser-Feshbach model for pre-equilibrium and evaporation processes. Note that the NBU and TR reactions were referred to as the stripping reactions to the continuum and bound states, respectively, in our past works [17-20,37-39].

The proposed method was applied to the ${ }^{7} \mathrm{Li}(d, x n)$ reaction at $40 \mathrm{MeV}$ for the first time [38], and then to the inclusive $(d, x p)$ reactions measured systematically for ${ }^{9} \mathrm{Be},{ }^{12} \mathrm{C},{ }^{27} \mathrm{Al},{ }^{58} \mathrm{Ni},{ }^{93} \mathrm{Nb},{ }^{181} \mathrm{Ta},{ }^{208} \mathrm{~Pb}$, and ${ }^{238} \mathrm{U}$ at $100 \mathrm{MeV}$ [39]. It should be noted that a phenomenological moving source model for pre-equilibrium and evaporation processes was used and single-nucleon TR reaction to bound states in the residual nuclei was ignored in Refs. [37-39]. These earlier studies encouraged us to develop an integrated code system for nuclear data evaluation of deuteron-induced reactions. As a result, a code system called DEURACS [17-20] has been developed by combining additional codes: CCONE [40] for preequilibrium and evaporation processes and DWUCK4 [41] for nucleon TR to bound states.

Typical results of the ${ }^{27} \mathrm{Al}(d, x p)$ reactions at 56 and $100 \mathrm{MeV}$ are shown in Fig. 5. The calculation reproduces fairly well both the shape and magnitude of the experimental $(d, x p)$ spectra including the peak structure observed in the high emission energy range, which is formed by single-neutron transfer ( $n$-TR) to bound states. The NBU process described by the Gauber model is a predominant $(d, x p)$ mechanism leading to the characteristic broad peak seen at half the incident energy.

Figure 6 show comparisons between the calculated and experimental TTNYs on ${ }^{9} \mathrm{Be}$ targets at angles around $0^{\circ}$ [17]. The calculation reproduces both the shape and
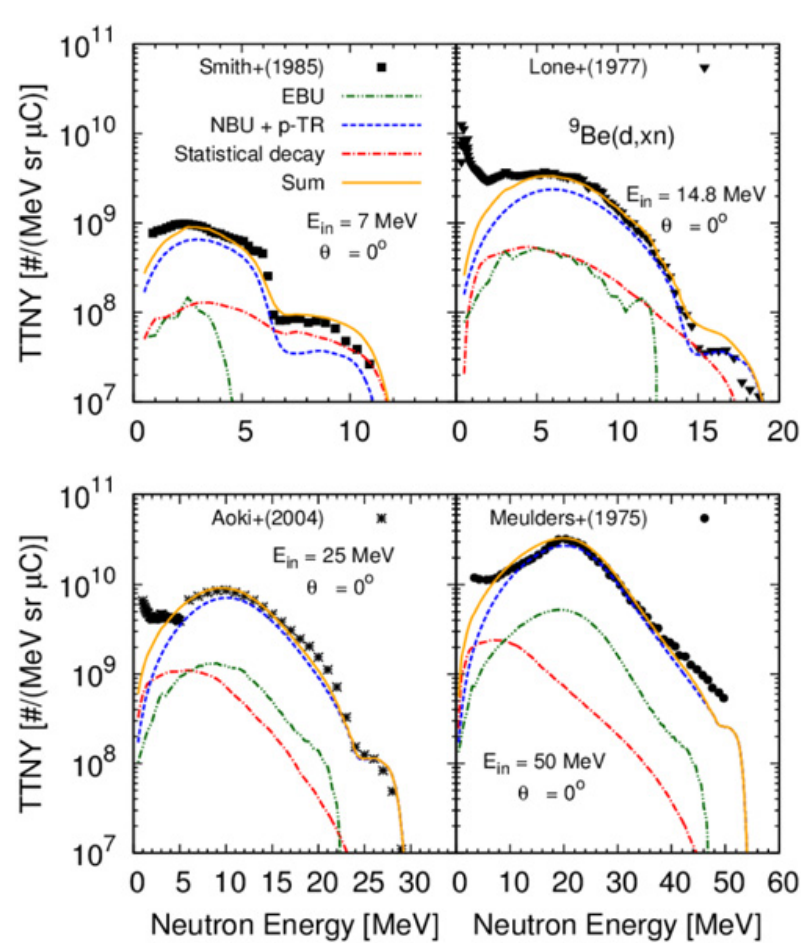

Figure 6. Calculated and experimental TTNYs for the ${ }^{9} \mathrm{Be}(d, x n)$ reaction at $0^{\circ}$. The dash-dot-dotted, the short-dashed, and the dash-dotted curves represent EBU, NBU + proton TR, and statistical decay, respectively. The solid curves are the sum of each component.

magnitude of the experimental TTNY data in the wide incident energy range up to $50 \mathrm{MeV}$. As shown in the figure, the NBU process is found to be the most dominant at any incident energies.

In the present TTNY calculation, we have adopted the semiclassical Glauber model to describe the NBU reaction. The bump structure seen around half the incident energy is described well by the Glauber model calculation in Fig. 6. Thus, the Glauber model seems to work well even at low incident energies although the model is based on the eikonal and adiabatic approximations. Our latest results of the $(d, x n)$ reaction at higher energy of $102 \mathrm{MeV}$ are given in Refs. [20,35].

In Fig. 6, a characteristic step-like structure is seen apparently in the high emission energy region at incident energies below $25 \mathrm{MeV}$. This high-energy component is formed by single-proton transfer reaction to discrete levels in the residual nuclei. This component is described by the DWBA calculation with the incident energy dependent spectroscopic factors [17] instead of the Glauber model calculation. Therefore, the DWBA calculation plays an essential role in reproducing neutron emission spectra in the high emission energy region, particularly for relatively low incident energies.

For the design of deuteron accelerator neutron sources, it is also important to estimate production of radioactive nuclei. DEURACS was successfully applied to calculations of some excitation functions of the $(d, p)$ reactions in Refs. $[18,43]$. Fig. 7 shows one of the results for the ${ }^{45} \mathrm{Sc}(d, p){ }^{46} \mathrm{Sc}$ reaction. We have found it essentially important to take proper account of the single-neutron transfer reaction to bound states in order to reproduce the experimental data as the incident energy increases. 


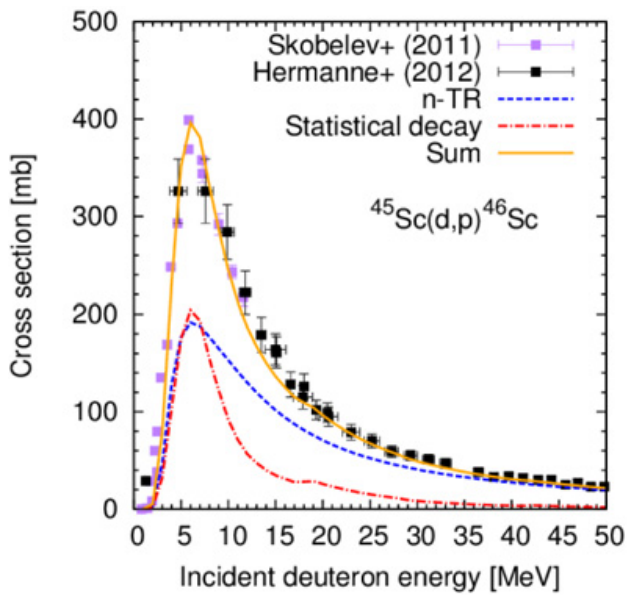

Figure 7. Calculated and experimental activation cross sections from the ${ }^{45} \mathrm{Sc}(d, p){ }^{46} \mathrm{Sc}$ reaction. The dashed and the dash-dotted curves denote the neutron TR and statistical decay, respectively. The solid curve is the sum of all components.

Since we have confirmed that DEURACS is successfully applicable to deuteron-induced reactions, we plan to perform nuclear data evaluation for neutron converter elements such as $\mathrm{Li}, \mathrm{Be}$, and $\mathrm{C}$ using available experimental data and DEURACS as a next step. A prototype nuclear data library will be produced for Monte Carlo transport codes such as PHITS and MCNPX in the future.

\section{Medical radioisotopes production}

A new production method has recently been proposed for medical radioisotopes production with accelerator neutrons by deuterons (GRAND) [1], aiming mainly at production of ${ }^{99} \mathrm{Mo}$. In the GRAND project, the $\mathrm{C}(d, x n)$ reaction is a candidate reaction as a neutron source. One of the authors has an interest in the generation of ${ }^{64} \mathrm{Cu}\left(\mathrm{T}_{1 / 2}=12.7 \mathrm{~h}\right)$ with accelerator neutrons by deuterons from the needs for a longer half-life PET radionuclide to diagnose the dynamics of a medicine in living body (cf. ${ }^{18} \mathrm{~F}: \mathrm{T}_{1 / 2}=1.8 \mathrm{~h}$ ) [44]. The radioisotope ${ }^{64} \mathrm{Cu}$ is a promising radionuclide suitable for labelling many radiopharmaceuticals for PET imaging because it decays by positron emission with its maximum energy of $0.653 \mathrm{MeV}$.

To generate ${ }^{64} \mathrm{Cu}$ of high isotopic purity, the ${ }^{64} \mathrm{Zn}(n, p){ }^{64} \mathrm{Cu}$ reaction at energies below $10 \mathrm{MeV}$ is promising because such by-products as ${ }^{63} \mathrm{Cu}$ and ${ }^{65} \mathrm{Cu}$ via the other reactions can be suppressed. We have performed a test experiment using mono-energetic neutron source based on the $\mathrm{D}(d, n)$ reaction for validation of the evaluated production cross sections at KUTL. In addition, an experiment was carried out for generation of ${ }^{64} \mathrm{Cu}$ from a natural $\mathrm{Zn}$ target using a prototype neutron source with the $\mathrm{C}(d, x n)$ reaction at KUTL, in order to optimize the incident deuteron energy and the design of $\mathrm{Zn}$ target. The result has been reported in Ref. [45].

More recently, two of the authors have proposed to produce ${ }^{92} \mathrm{Y}$ using the $\mathrm{C}(d, x n)$ neutrons. It is an alternative of ${ }^{111} \mathrm{In}$ that is used for bioscan before radioimmunotherapy of ${ }^{90} \mathrm{Y}$ ibritumomab tiuxetan. ${ }^{92} \mathrm{Y}$ is produced via the ${ }^{92} \mathrm{Zr}(n, p)$ reaction. A feasibility experiment was performed at Cyclotron and Radioisotope
Center (CYRIC), Tohoku University. A carbon thick target was irradiated by $20-\mathrm{MeV}$ deuterons to generate accelerator neutrons. The thick target neutron yield (TTNY) was measured by using the multiple foils activation method. The amount of ${ }^{92} \mathrm{Y}$ production and induced impurities were estimated by simulation with the measured TTNY and neutron nuclear data [46]. The detail of the experimental result is reported in Ref. [47].

\section{Summary and outlook}

A comprehensive research program on deuteron nuclear data for the design of accelerator-based neutron sources was reviewed. The program consists of nuclear data measurements including thick target neutron and gamma yields, theoretical model analyses and code development, cross section evaluation and benchmark test, and application to medical radioisotopes production. Toward our goal to develop a state-of-the-art nuclear data library up to $200 \mathrm{MeV}$, the following actions will be taken in the future:

- Continued measurements of neutron and gamma-ray production from deuteron-induced reactions at incident energies up to $200 \mathrm{MeV}$.

- Validation of DEURACS using a variety of differential data and its improvement.

- Cross section evaluation and production of a prototype nuclear data library for specific nuclei (e.g., Li, Be, and C) contained in neutron converters.

- Benchmark testing by Monte Carlo transport codes (e.g., PHITS) with newly-evaluated nuclear data library using experimental thick target neutron yields.

- Application of deuteron transport calculation method to the design of medical radioisotopes production.

The authors wish to thank all co-workers for their kind cooperation and fruitful discussion: N. Shigyo, K. Sagara, S. Maebara, H. Takahashi, H. Sakaki, Y. Tajiri, and K. Hirabayashi for TTY measurements at KUTL; Y. Iwamoto, D. Satoh, M. Hagiwara, H. Yashima, T. Shima, M. Kitajima, K. Nakano, and H. Sadamatsu for DDX measurements at RCNP; K. Ogata and T. Ye for theoretical model and code development; M. Ito, Y. Sanzen, and M. Kamida for RI production experiments at CYRIC.

\section{References}

[1] Y. Nagai et al., J. of Phys. Soc. of Japan, 82, 064201 (2013)

[2] S. Agosteo, G. Curzio, F. d'Errico, et al., Nucl. Instr. Meth. in Phys. Res. A 476, 106 (2002)

[3] A. Moeslang, V. Heinzel, H. Matsui, M. Sugimoto, Fusion Eng. Des. 81, 863 (2006)

[4] M. Fadil, B. Rannou, Nucl. Inst. Method. in Phys. Res. B 266, 4318 (2008)

[5] Implication of Partitioning and Transmutation in Radioactive Waste Management IAEA Technical Reports Series No. 435 (2004); R. Fujita, J. of Atomic Energy Soc. of Japan, 58(2), 28 (2016) (in Japanese)

[6] Y. Uwamino, T. Ohkubo, Nucl. Instr. Meth. in Phys. Res. A 271, 546-552 (1988)

[7] M.J. Saltmarsh, C.A., Ludemann, C.B., Fulmer, R.C. Styles, Nucl. Instr. Meth. 145, 81-90 (1977) 
[8] H.J. Brede, G. Dietze, K. Kudo, et al., Nucl. Instr. Meth. in Phys. Res. A 274, 332-344 (1989)

[9] TENDL [internet]. Available from: https:// tendl.web.psi.ch/tendl_2015/tendl2015. html

[10] Forrest R, Capote R, Otsuka, et al., FENDL3 Library Summary documentation, International Atomic Energy Agency, Nuclear Data Section; 2012. (INDC(NDS)-0628)

[11] A.J. Koning, D. Rochman, Nucl. Data Sheets 113, $2841(2012)$

[12] P. Sauvan, A. Mayoral, J. Sanz, et al., J. of the Korean Phys. Soc. 59(2), 1195-1198, (2011)

[13] EXFOR [internet]. Available from: https://wwwnds.iaea.org/exfor/exfor.htm

[14] Jin Lei, A.M. Moro, Phy. Rev. C 92, 044616 (2015)

[15] G. Potel, F. M. Nunes, I.J. Tompson, Phy. Rev. C 92, 034611 (2015)

[16] B.V. Carlson, R. Capote, M. Sin, Few-Body Syst. 57, 307 (2016)

[17] S. Nakayama, H. Kouno, Y. Watanabe, et al., Phys. Rev. C 94, 014618 (2016)

[18] S. Nakayama, Y. Watanabe, J. Nucl. Sci. Technol. 53, 89 (2016)

[19] S. Nakayama, S. Araki, Y.Watanabe, et al., Nucl. Data Sheets 118, 305 (2014)

[20] S. Nakayama, H. Kouno, Y. Watanabe, et al., presented at this conference (2016)

[21] M, Avrigeanu, E. Simeckova, U. Fischer, et al., Phy. Rev. C 94, 014606 (2016) and references therein

[22] A. Boudard, J. Cugnon, J.-C. David, et al., Phys. Rev. C87, 014606 (2013)

[23] T. Sato, K. Niita, N. Matsuda, et al., J. Nucl. Sci. Technol. 50, 913 (2013)

[24] S. Hashimoto, Y. Iwamoto, T. Sato, et al., Nucl. Instr. Meth. B 333, 27 (2014)

[25] N. Shigyo, K. Hidaka, K. Hirabayashi, et al., J. Kor. Phys. Soc. 59(2), 1725-1728 (2011)

[26] K. Hirabayashi, T. Nishizawa, H. Uehara et al., Prog. in Nucl. Sci. and Technol. 3, 60-64 (2012)

[27] Y. Tajiri, Y, Watanabe, N. Shigyo, et al., Prog. in Nucl. Sci. and Technol. 4, 582-586 (2014)
[28] S. Araki, Y. Watanabe, T. Kin, et al., Energy Procedia 71, 197-204 (2015)

[29] S. Araki, Y. Watanabe, T. Kin, et al., Ionizaing Radiation 41(3), 127-132 (2016) (in Japanese)

[30] S. Araki, K. Kondo, T. Kin, Y. Watanabe, N. Shigyo, K. Sagara, Proc. of ISORD-8 (to be published)

[31] R.H. Johnson, D.T. Ingersoll, B.W. Wehring, J.J. Dorning, Nucl. Instr. Meth. 145, 337-346 (1977)

[32] D. Satoh, T. Sato, N. Shigyo, K. Ishibashi, JAEAData/Code 2006-023, Japan Atomic Energy Research Institute (2006)

[33] S. Furihata, Nucl. Instrum. Methods B 171, 251-258 (2000)

[34] T. Ogawa et al., Nucl. Instrum. Methods B 325, 35 (2014)

[35] S. Araki, Y. Watanabe, M. Kitajima, et al., presented at this conference (2016)

[36] K. Iida, A. Kohama, K. Oyamatsu, J. of the Phys. Soc. of Jpn 76(4), 044201 (2007)

[37] T. Ye, Y. Watanabe, K. Ogata, Phys. Rev. C 80, 014604 (2009)

[38] T. Ye, S. Hashimoto, Y. Watanabe, K. Ogata, M. Yahiro, Phys. Rev. C 84, 054606 (2011)

[39] T. Ye, S. Hashimoto, Y. Watanabe, et al., , J. Kor. Phys. Soc. 59(2), 871-874 (2011)

[40] O. Iwamoto, J. Nucl. Sci. Technol. 44(5), 687-697 (2007); O. Iwamoto et al., Nuclear Data Sheets 131, 259-288 (2016)

[41] P.D. Kunz, DWUCK4 (1974)

[42] S. Nakayama, S. Araki, Y. Watanabe, et al., Energy Procedia 71, 219 (2015)

[43] S. Nakayama, and Y. Watanabe, JAEA-Conf 2015003, 105 (2015)

[44] T. Kin, Y. Nagai, N. Iwamoto, et al., J. of Phys. Soc. of Jpn. 82(3), 034201 (2013)

[45] T. Kawagoe, T. Kin, S. Araki, Y. Watanabe, JAEAConf 2015-003, 297 (2015)

[46] K. Shibata et al., J. Nucl. Sci. Technol. 48(1), 1-30 (2011)

[47] T. Kin Y. Sanzen, M. Kamida, et al., presented at this conference (2016) 